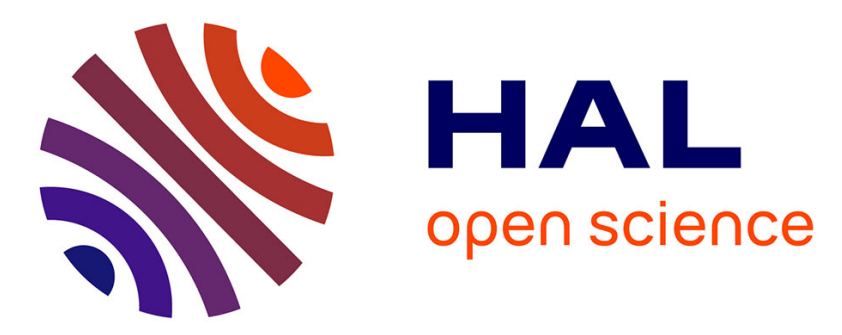

\title{
ELECTRON SPIN POLARIZATION : A NEW TOOL IN PHOTOEMISSION AND MAGNETISM
}

\author{
M. Campagna, D. Pierce, K. Sattler, H. Siegmann
}

\section{To cite this version:}

M. Campagna, D. Pierce, K. Sattler, H. Siegmann. ELECTRON SPIN POLARIZATION : A NEW TOOL IN PHOTOEMISSION AND MAGNETISM. Journal de Physique Colloques, 1973, 34 (C6), pp.C6-87-C6-94. 10.1051/jphyscol:1973623 . jpa-00215344

\section{HAL Id: jpa-00215344 https://hal.science/jpa-00215344}

Submitted on 1 Jan 1973

HAL is a multi-disciplinary open access archive for the deposit and dissemination of scientific research documents, whether they are published or not. The documents may come from teaching and research institutions in France or abroad, or from public or private research centers.
L'archive ouverte pluridisciplinaire HAL, est destinée au dépôt et à la diffusion de documents scientifiques de niveau recherche, publiés ou non, émanant des établissements d'enseignement et de recherche français ou étrangers, des laboratoires publics ou privés. 


\title{
ELECTRON SPIN POLARIZATION : A NEW TOOL IN PHOTOEMISSION AND MAGNETISM
}

\author{
M. CAMPAGNA, D. T. PIERCE, K. SATTLER and H. C. SIEGMANN
}

Laboratorium für Festkörperphysik ETHZ, Hönggerberg, CH-8049 Zürich, Switzerland

\begin{abstract}
Résumé. - On passe en revue la technique permettant de mesurer la polarisation du spin $P$ des électrons émis par effet photoélectrique et on présente des résultats obtenus pour différents matériaux magnétiques. Les courbes de magnétisation photoélectrique s'obtiennent en mesurant $P$ en fonction du champ magnétique appliqué tandis que la mesure de $P$ en fonction de l'énergie des photons livre un spectre de la polarisation du spin qui permet de localiser les états magnétiques dans un schéma des niveaux d'énergie. Les valeurs positives de $P$ observées pour $\mathrm{Ni}$ et Co sont en désaccord avec les prédictions s'appuyant sur le modèle de bande du ferromagnétisme. Dans les semiconducteurs désordonnés apparaissent dans la zone interdite de nouveaux états qui sont occupés par un seul électron et qui renforcent le coupiage ferromagnétique des spins localisés des états $4 \mathrm{f}$. La présence d'une couche magnétique non saturée a été trouvée à la surface d'un monocristal ferromagnétique tel que EuO. Cette couche morte est à l'origine d'une diffraction d'échange du spin pour les électrons provenant de l'intérieur ferromagnétique du cristal. Le dopage avec des ions métalliques trivalents a pour effet d'augmenter $P$, car les électrons supplémentaires renforcent le champ moléculaire dans la couche superficielle paramagnétique.
\end{abstract}

Abstract. - A review of the technique of measuring the spin polarization $P$ of photoemitted electrons and of results of measurements from a variety of magnetic materials is presented. Photoelectric magnetization curves are obtained by measuring $P$ as a function of applied magnetic field, while measuring $P$ as a function of photon energy gives a spectrum of spin polarization which locates magnetic states in an energy level scheme. The positive $P$ observed for $\mathrm{Ni}$ and $\mathrm{Co}$ is in disagreement with the predictions of the band model of ferromagnetism. On disordering, magnetic semiconductors exhibit new states in the gap which are found to be singly occupied and to enhance the ferromagnetic coupling of the localized $4 \mathrm{f}$ spins. At the single crystal surface of the Heisenberg ferromagnet EuO a paramagnetic sheet was found to exist. This dead layer causes spin exchange scattering for electrons emitted from the ferromagnetic bulk. $P$ is found to increase on doping with trivalent metal ions : excess electrons enhance the molecular field in the paramagnetic surface sheet.

1. Photoelectrons from magnetic materials exhibit electron spin polarization (ESP). - - If the spindirection is not severely affected in the process of photoemission, electrons emitted from magnetic materials must show a spin polarization. Conservation of the expectation value of spin direction is usually to be expected in photoemission, because the emission processes are fast compared to the spin relaxation time.

The degree of spin polarization is expected to reflect the magnetization of the initial state from which the electron was emitted. The measurement of the degree of spin polarization of electrons from different electron states may then yield information on the energy distribution of magnetization.

The ESP is defined as the expectation value of the pauli spin operator $\sigma$, and for a given direction in space, say the $z$-direction, one obtains for the degree of ESP along $z$ :

$$
<\sigma_{z}>=P=\frac{n \uparrow-n \downarrow}{n \uparrow+n \downarrow}
$$

where $n \uparrow, n \downarrow$ are the respective numbers of up and down spin electrons (magnetic moment respectively parallel and antiparallel to the magnetic field direction).

2. The apparatus consists of a photoemitter in a homogeneous magnetic field and ultrahigh vacuum, an electron accelerator system and a Mott scatterer as analyser for the polarization. - The principle of the experiments observing the emission of polarized electrons from magnetic cathodes is shown in figure 1. The cathode is a magnetic material in which a magnetization $\mathbf{M}$ perpendicular to the emitting surface is generated by an external magnetic field. Electrons are emitted by irradiating with ultraviolet light. The emitted electrons are replaced by electrons from an unpolarized electron reservoir that keeps the cathode at constant electric potential. There is a flow of electrons from the reservoir through the cathode into vacuum: the magnetic cathode acts as the polarizing spin filter. Electrostatic deflection converts the longitudinal polarization into a transverse one. The right-left asymmetry in the scattering from a gold foil detects the degree of spin polarization parallel to the direction of the magnetization. 


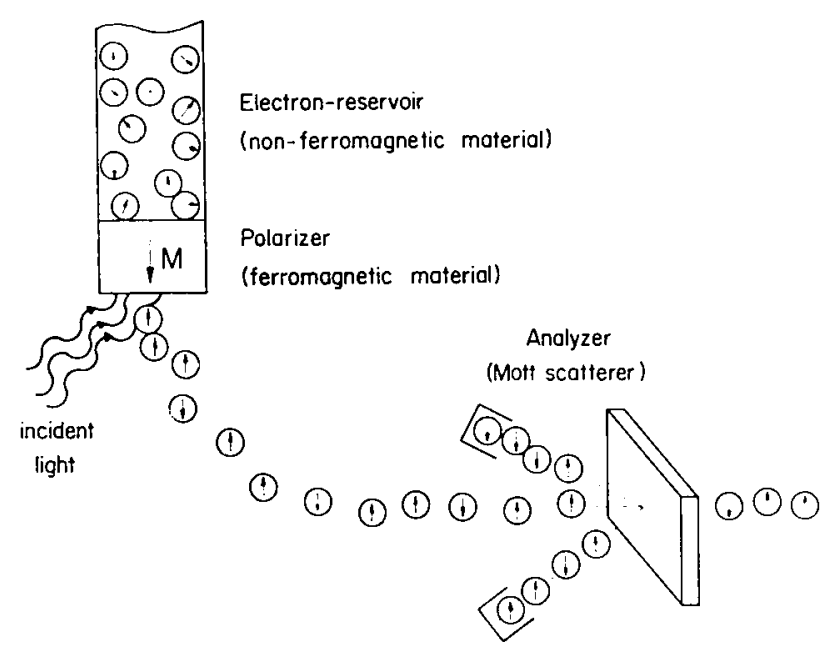

FIG. 1. - The principle of the experiment (see text).

Mott [1] first pointed out in 1929 that elastic Coulomb scattering of electrons from heavy atoms depends on the spin state of the electrons through LS coupling on scattering. Figure 2 shows the configuration for Mott scattering. Electrons which have been accelerated to $100 \mathrm{keV}$ are scattered from a gold foil which is thin to minimize multiple scattering. The intensities $\left(N_{1}, N_{2}\right)$ are measured at scattering angles $\pm 120^{\circ}$. The polarization is related to the measured asymmetry $A$ by

$$
A=\left(A_{0}+P S\right) /\left(1+A_{0} P S\right) .
$$

By reversing the magnetic field at the sample we can measure and eliminate the effect of the apparatus asymmetry $A_{0}$, which arises from slight asymmetries in the sensitivity and orientation of the detectors [2]. The Sherman function $S$ is well known from theoretical calculations which have been experimentally verified [3].

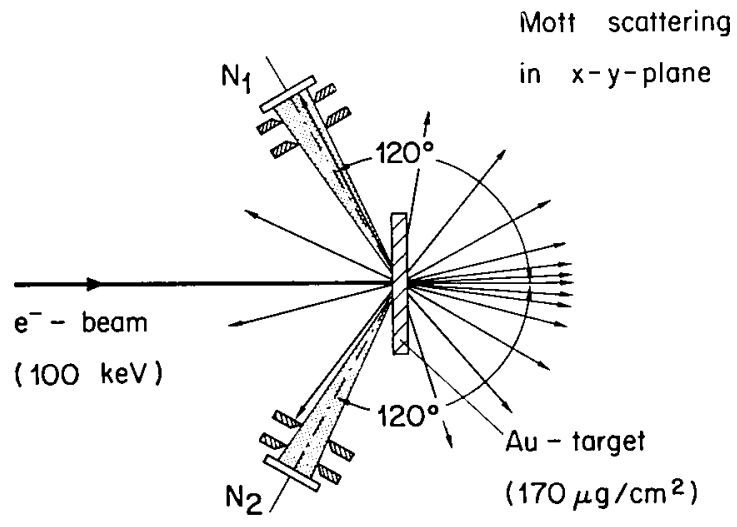

$$
A=\frac{N_{1}-N_{2}}{N_{1}+N_{2}}, \quad S=0,31, P=\frac{1}{S} A
$$

FIG. 2. - Measurement of electron spin polarization (ESP) by Mott scattering. $A$ is the observed scattering asymmetry which is related to the degree $P$ of ESP along the $z$-direction.
A schematic diagram of the actual apparatus for observing electron polarization in photoemission is shown in figure 3 . The surface of the sample must be atomically clean and therefore is in ultrahigh vacuum (UHV), typically $1 \times 10^{-10}$ torr. Measurements have been performed on thin film samples evaporated in situ and on single crystals cleaved in UHV. The light source used is a $\mathrm{Hg}-\mathrm{Xe}$ high pressure arc lamp with photon energies up to about $6 \mathrm{eV}$.

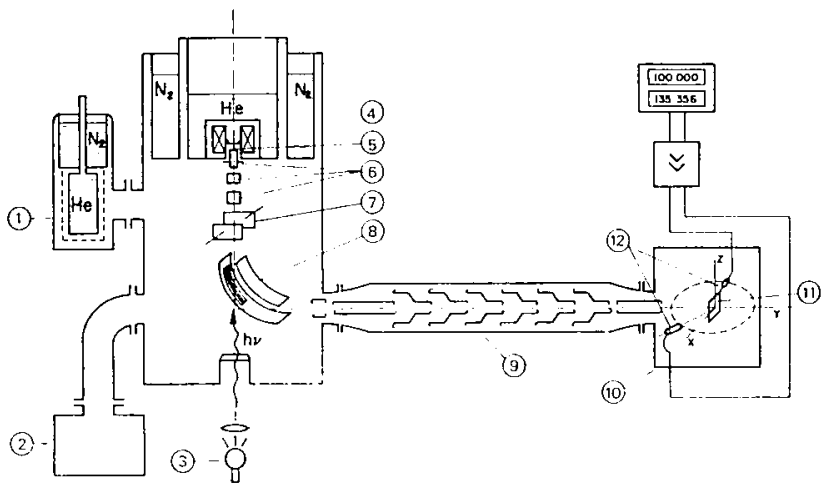

FIG. 3. - Schematic diagram of the apparatus for detection of ESP in photoemission. The main parts are : 1) cryopump, 2) ion pump, 3) $\mathrm{Hg}-\mathrm{Xe}$ arc lamp, 4) superconducting magnet, 5) sample, 6) accelerating electrodes, 7) planar condenser, 8) cylindrical condenser to deflect electrons, 9) accelerator, 10) Mott scatterer, 11) gold foil, 12) counters.

3. The measurements include photoelectric magnetization curves (PMC), spectra of spin polarization (SSP), and (spin independent) quantum yield. Measurement of the degree of spin polarization of the photoemitted electrons as a function of magnetic field keeping photon energy $\hbar \omega$ and temperature $T$ constant is analogous to a magnetization measurement $M$ vs. $H$ and therefore has been named a photoelectric magnetization curve.

If the magnetic field is held constant and $T$ is constant, one obtains the spectra of spin polarization. The spectra contain information on the spin polarization of the different electron states since as the photon energy is increased, electrons from the next deeper lying energy states are also photoemitted.

The quantum yield $Y$ is the number of electrons photoemitted per incident photon as a function of $\hbar \omega$. The threshold photon energy $\varphi$ at which electron emission sets in is the energy difference between the first occupied electron state and the vacuum level $E_{\infty}$. The yield measurement gives the total photocurrent at each photon energy, whereas the SSP gives the polarization of this current.

Other types of ESP measurements are also possible. Measurement of the spin polarization as a function of temperature $P(T)$ at constant $H$ and $\hbar \omega$ is especially interesting as the ordering temperature is approached. By measuring the kinetic energy of the photoemitted electrons in addition to the spin, one could obtain $P$ as a function of initial state energy of the electron, 
that is, an energy distribution of spin polarization $P(E)$. Measurements of $P(T)$ are in progress, but several experimental difficulties, particularly the high applied field magnetic at the sample, relegate measurements of $P(E)$ to the future.

4. PMC's are related to magnetization curves. - In most magnetic substances, the magnetization is produced predominantly by the spin moment of the electrons. Thus the measured polarization is proportional to the $z$ component of the magnetization :

$$
P=f(h \omega) M(H, T) / M_{0}
$$

where $M_{0}$ is the magnetization when all the spins are aligned. (In the case of EuO, special surface effects are present and this relation does not hold, see 10.) The dimensionless factor $f(\hbar \omega)$ depends on photon energy and denotes the relative contribution to the photoelectric current from magnetic and non-magnetic (doubly occupied) states, $0 \leqslant f \leqslant 1$. The photoelectric magnetization measurement is obtained from a very small amount of the substance given by the approximately $1 \mathrm{~mm}^{2}$ effective photocathode area and the escape length of the photoelectrons which is $10-30 \AA$ for metals and up to about $100 \AA$ for semjconductors. Figures 4,5 and 6 show examples of PMC from ferromagnetic [4], antiferromagnetic [5] and paramagnetic [5] substances at a temperature of $4.2 \mathrm{~K}$.

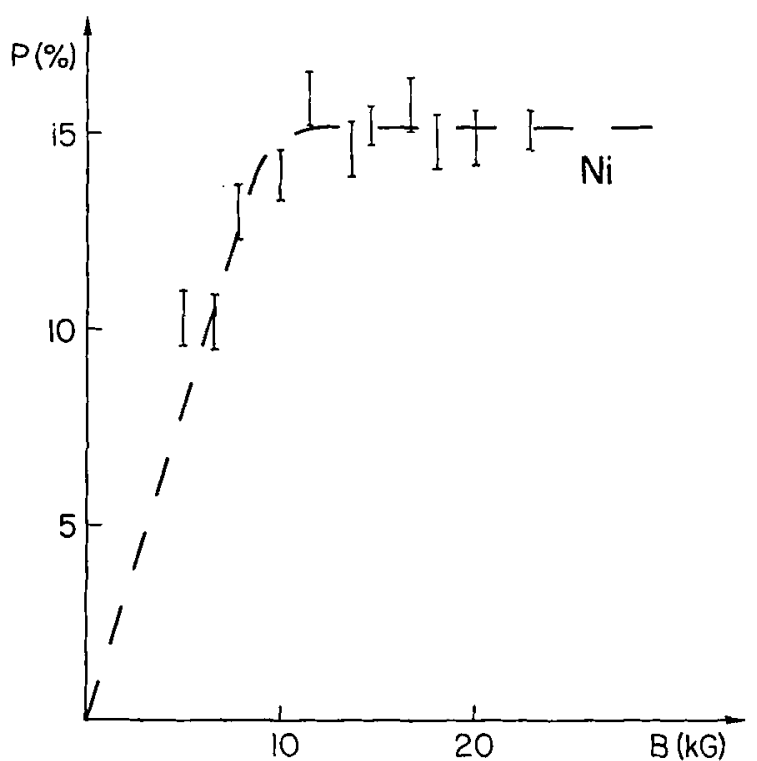

FIG. 4. - Photoelectric Magnetization Curve (PMC) for ferromagnetic Ni taking the full spectrum of the lamp. $\varphi(\mathrm{Ni})=5.0 \mathrm{eV}$, $\hbar \omega=5-6 \mathrm{eV}$.

The PMC in figure 4 is for a polycrystalline $\mathrm{Ni}$ film [4]. The polarization increases sharply and saturates. This behavior is expected for a magnetization curve of a ferromagnetic thin film with magnetic field perpendicular to the film. Since the demagne-

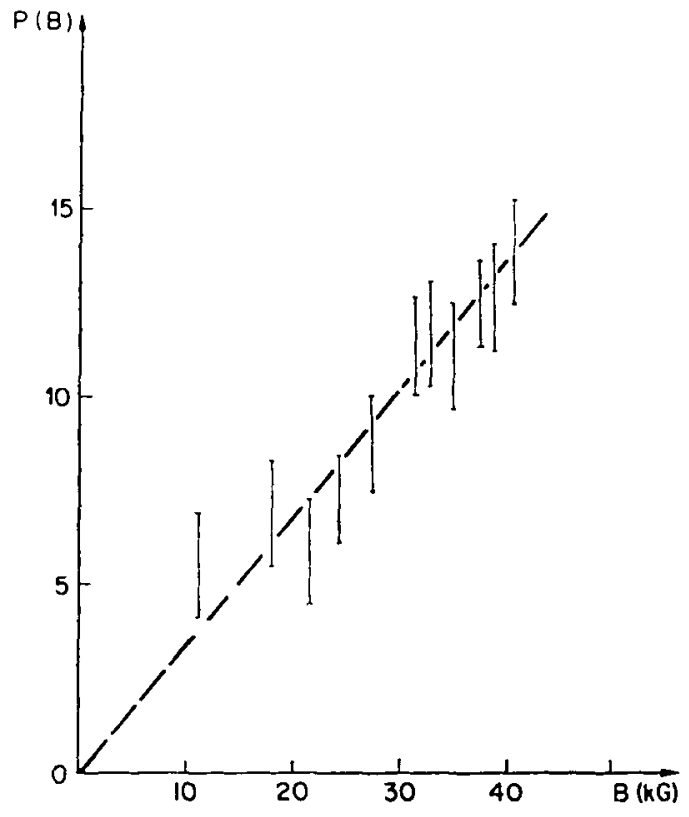

FIG. 5. - PMC for antiferromagnetic GdP. The measurement temperature is $4.2 \mathrm{~K}$. The curve was taken using the full spectrum of the lamp.

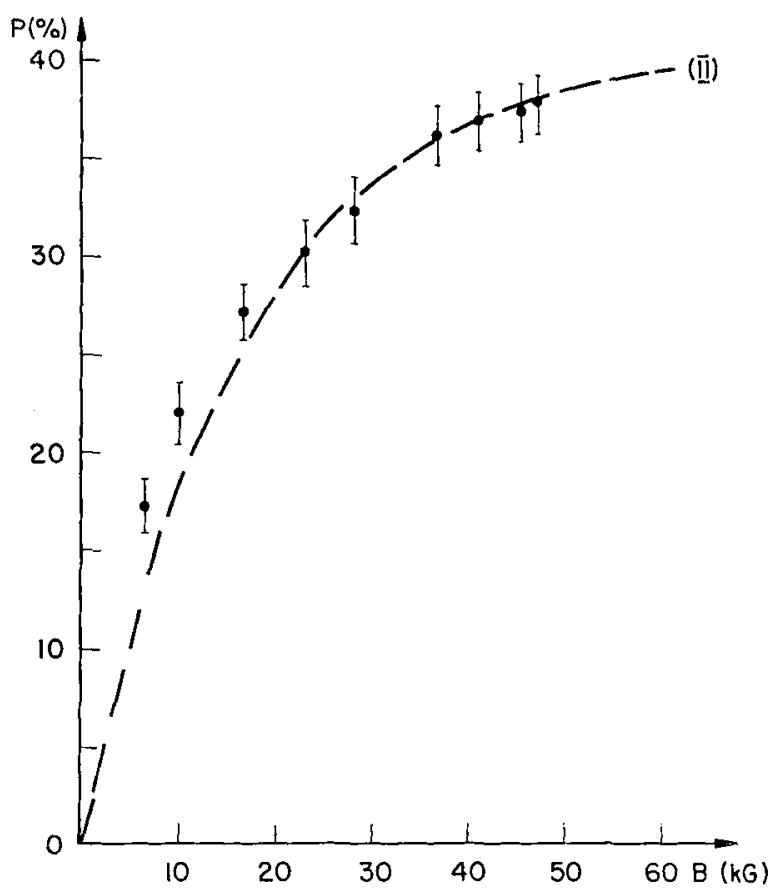

FlG. 6. - PMC of paramagnetic GdP obtained by evaparating onto cold substrate $(T=4.2 \mathrm{~K}, \hbar \omega=3.92 \mathrm{eV})$. The dashed line is a Brillouin function.

tizing field is maximum in this confirmation, a field about equal to the saturation magnetization $M_{\mathrm{s}} \simeq 6 \mathrm{kG}$ must be applied in order to align the Weiss domains.

Figure 5 shows a PMC for GdP [5] which is antiferromagnetic with a Neel temperature of $15 \mathrm{~K}$. The induced magnetization curve of bulk GdP at $4.2 \mathrm{~K}$ is linear and ferromagnetic saturation occurs at $90 \mathrm{kOe}$. As expected, the PMC can also be fitted 
by a straight line within the statistical uncertainty. The observed photoelectrons are not emitted from the $4 \mathrm{f}$ shells but from higher lying energy states. These states are so strongly coupled by the exchange field to the $4 \mathrm{f}$ shells that they reflect the magnetization state of the $4 f$ electrons.

Although $\mathrm{GdP}$ is an antiferromagnet with $\mathrm{NaCl}$ structure, when it is evaporated onto a substrate at $4.2 \mathrm{~K}$ the structure is so disturbed that the antiferromagnetic coupling is broken and paramagnetic properties are displayed [5]. The dashed line in figure 6 is a Brillouin function for 7 Bohr magnetons, corresponding to the ${ }^{8} \mathrm{~S}_{7 / 2}$ configuration of the half filled 4f shell of the Gd ion.

5. SSP are consistent with optical absorption and intensity photoemission studies and in addition allow a location of the magnetic states in an energy level scheme. - Consider, for example, the SSP of the ferromagnetic semiconductor $\mathrm{Eu}_{3} \mathrm{P}_{2}$ [6] shown in figure 7. In $\mathrm{Eu}_{3} \mathrm{P}_{2}$ as in most magnetic semiconductors there are three types of levels: magnetic localized levels, conduction or impurity states, and the full valence bands. The ESP of electrons from the $4 \mathrm{f}$-states is equal to the relative magnetization.

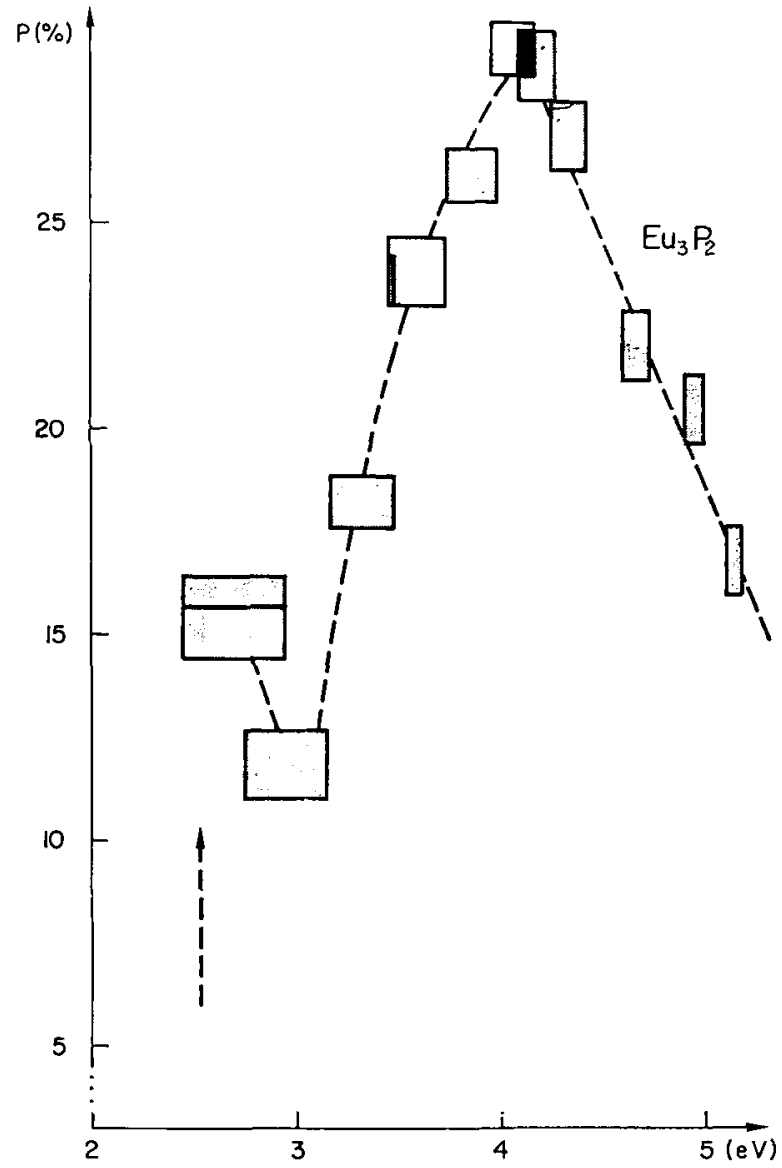

Fig. 7. - Spectrum of Spin Polarization (SSP) of $\mathrm{Eu}_{3} \mathrm{P}_{2}$ at $T=4.2 \mathrm{~K}$ and at $H=22.8 \mathrm{kG}$. The rectangles represent the experimental results with the resolution of the monochromator (horizontal width) and the statistical uncertainty in the measurement of the ESP (vertical width).
The conduction - and impurity - states are also polarized, but often to a lesser extent because of admixture of doubly occupied states. The electrons from the doubly occupied valence bands are not spin polarized. The SSP show at which photon energy the emission from these states sets in. In figure 7 , at low photon energies near the photothreshold, we have emission from impurity states. The increase of the polarization on increasing the photon energy indicates the onset of emission from $4 \mathrm{f}^{7}$. states. The decrease is due to the emission of unpolarized valence band electrons. These measurements enable us to make assignments of the transitions observed in optical absorption [7] and help to undertand the energy distribution curves of photoelectrons.

It is clearly possible to distinguish between the 3 types of levels. However, if one wants to locate the levels say with respect to the vacuum level, the one electron approximation must be valid or the energy of the hole left behind must be known as a function of photon energy and other possible parameters. This difficulty is of course present in all spectroscopic methods.

6. For photon energies a few $\mathrm{eV}$ above threshold, the SSP for $\mathrm{Fe}, \mathrm{Co}$ and $\mathrm{Ni}$ tend to the expected asymptotic values, but, unexpectedly, the shape of the SSP is similar for the three metals and $P$ is high only for electrons near $E_{\mathrm{F}}$. $-\mathrm{SSP}$ for $\mathrm{Fe}, \mathrm{Co}$ and $\mathrm{Ni}[8]$ are shown in figure 8 . The work function was reduced

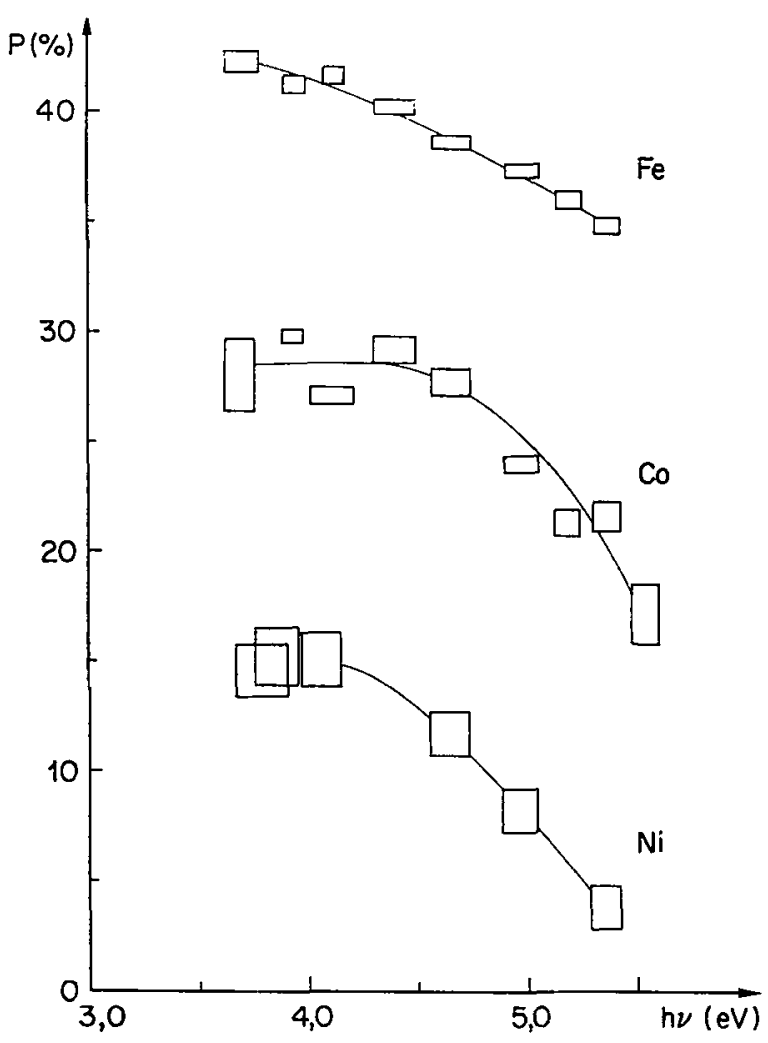

Fig. 8. - SSP for Fe, Co and Ni. Light cesiation was used to reduce the work function to $\sim 3.2 \mathrm{eV}$ for all three metals. The magnetic field is higher than the saturation field. 
from the values of the clean metals of $\sim 5 \mathrm{eV}$ by light cesiation to $3.2-3.3 \mathrm{eV}$. The covering parameter 0 is approximately 0.2 . The samples are thin films evaporated under ultra-high vacuum conditions onto a substrate heated to $\sim 400 \mathrm{~K}$. During the measurements, the temperature of the samples is around $10 \mathrm{~K}$.

The shape of the spectra is similar for all three metals : high ESP for electrons emitted with photon energies near the energy of the photoelectric threshold, and the ESP decreases on increasing the photon energy. The maximum value of the ESP decreases with increasing number of d-electrons. The spectral width of the region with high ESP also decreases on going from $\mathrm{Fe}$ to $\mathrm{Ni}$ and shows that the magnetism is generated predominantly by electron states within about one eV of the Fermi level.

Without employing any theory of magnetism we can make the following statement : if $n_{\mathrm{B}}$ is the number of Bohr magnetons and $n$ the total number of $d$ and s-electrons in the conduction bands, the spin polarization should tend towards the value $n_{\mathrm{B}} / n$ for $\hbar \omega \gg \varphi$. This arises because for high photon energies the escape function $T(E)$ is slowly varying and all electrons from the conduction bands can escape equally well from the material. It is also assumed that the ESP is conserved in photoemission and that $k$-conservation is not very important in the magnetic metals. We have $n_{\mathrm{B}} / n=+26 \%,+17 \%$ and $+5.5 \%$ for $\mathrm{Fe}, \mathrm{Co}$ and $\mathrm{Ni}$ respectively [9]. As seen in figure 8, the ESP tends towards these values.

7. The measured $\boldsymbol{P}$ for $\mathrm{Ni}$ and $\mathrm{Co}$ is always positive in contrast to expectations from the band model of ferromagnetism and current band calculations. In the band model of ferromagnetism, largely due to Stoner, Wohlfarth and Slater (SWS), the exchange between the itinerant electrons produces an average Hartree-Fock molecular field that is different for the two electron spin states. That is, electrons with the same wave vector $\mathbf{k}$ have different energy depending on their spin. The band is formally divided into two pure spin subbands which are split by the exchange splitting energy $\Delta E_{\text {ex }}$ thought to be about $0.4 \mathrm{eV}$ for $\mathrm{Ni}$ and $1.1 \mathrm{eV}$ for Co at $T=0 \mathrm{~K}$. For Co and Ni, one subband is filled (majority spins) while the other is only partially filled (minority spins) giving rise to the net magnetic moment. The top of the majority spin band lies below the Fermi level so that electrons excited near the Fermi level should be minority spin electrons and a negative polarization should be observed. The expected $P$ for Co calculated [10] on the basis of the nondirect transition model including escape probability is shown in figure 9. For curve $(a)$, the density of states from the band structure calculation of Wong et al. [11] was used $\left(\Delta E_{\mathrm{cx}}=1.35 \mathrm{eV}\right)$ and for curve $(b)$ the best value for the exchange splitting $\left(\Delta E_{\mathrm{cx}}=1.05 \mathrm{eV}\right)$ according to Wohlfarth [12] was used.

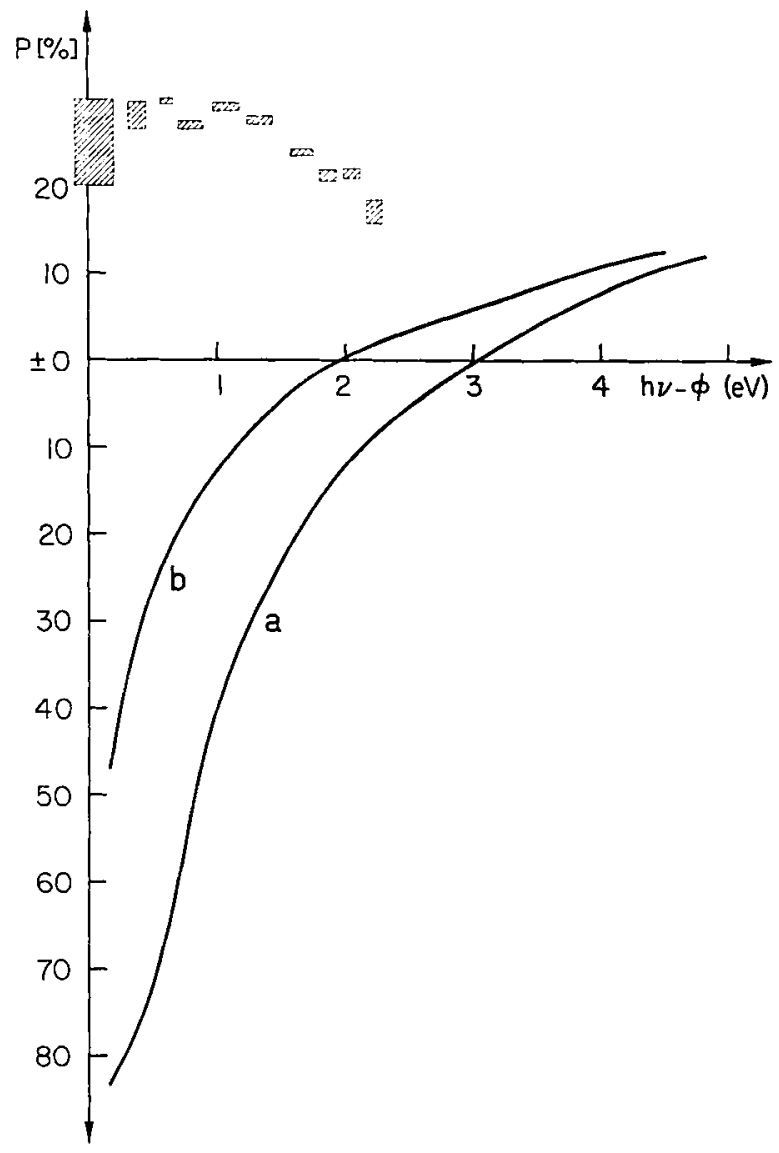

FIG. 9. - SSP of Co with the work function lowered by cesiation to $3.3 \mathrm{eV}$. Solid line lower curve predicted using the band structure of reference [11], upper curve with the best value of the exchange splitting suggested in reference [12].

A negative polarization is expected over a wide energy range but a positive polarization was always observed. In order to reduce the possibility that a negative $P$ was being obscured as a consequence of $\mathbf{k}$ conservation and a peculiarity in the band structure, the work function was varied over about $1 \mathrm{eV}$ using $\mathrm{Cs}$, thus varying also the initial and final states observed for a given photon energy [10]. That a positive $P$ is always observed suggests that the SWS band model, with current band calculations, is not sufficient to explain the ESP results.

It appears that many body effects may be crucial to magnetism in the $3 \mathrm{~d}$ transition metals, but calculations along these lines have not proceeded to the point of quantitative comparison with experiment [13]-[17]. Another possibility is that the hole left behind on photoemission of an electron may be strongly localized and single atom behavior may be dominant [18]. If the one electron approximation breaks down in this way, a comparison of the ESP measurements and the SWS band model does not make sense. The ESP experiments are important not only as a tool to investigate magnetism in solids but also the photoemission process itself. 
8. On disordering new localized states are observed in the gap of some magnetic semiconductors. EuS, EuSe, EuTe, GdP, GdAs and GdSb [5] were selected for investigating effects of structural disorder because (i) it is possible to prepare thin films by electron gun evaporation (ii) all materials have the $\mathrm{NaCl}$ lattice, and (iii) the forbidden energy zone $E_{\mathrm{GV}}$ between valence and conduction band states varies from $2,32 \mathrm{eV}$ in EuS to $\approx 0$ in GdSb. EuS and EuSe are ferromagnets while the other compounds are antiferromagnets. An energy level scheme for these substances is shown in figure 10 .

The systematic variation of the lattice constant, ordering temperatures and energy parameters are given in table I. Generally, the width $E_{\mathrm{GV}}$ of the forbidden energy zone decreases with decreasing ionicity of the bonds. The energy of the magnetic states $E_{\mathrm{G} 4 \mathrm{f}}$ is mainly determined by the Coulomb field of the ion $\left(\mathrm{Gd}^{++}\right.$or $\left.\mathrm{Eu}^{++}\right)$.

Much theoretical work shows that localized states in the energy gap are expected to appear on disordering [19]. The position of the $4 f^{7}$-states in the magnetic semiconductors remain largely unaffected on disordering due to their extreme localization and thus provide a point of reference. The difficulty with the ionic magnetic semiconductors is that they are not glassformers. However, it is possible to introduce structural disorder by evaporating thin films onto a substrate cooled to liquid He temperature. In the following discussions, annealed (ordered) samples are labelled 1 , and disordered II.

The photothreshold $\varphi$ was determined from yield measurements and found to decrease on disordering due to new states in the gap. We define

$$
\Delta \varphi=\varphi-\varphi^{*},
$$

where $\varphi^{*}$ is the photothreshold of the disordered state. $\Delta \varphi>0$ for all materials investigated. The change of $\varphi$ on disordering, $\Delta \varphi$, was found to be largest for EuS and decreases with decreasing $E_{\mathrm{GV}}$. For the interpretation it is important to note that

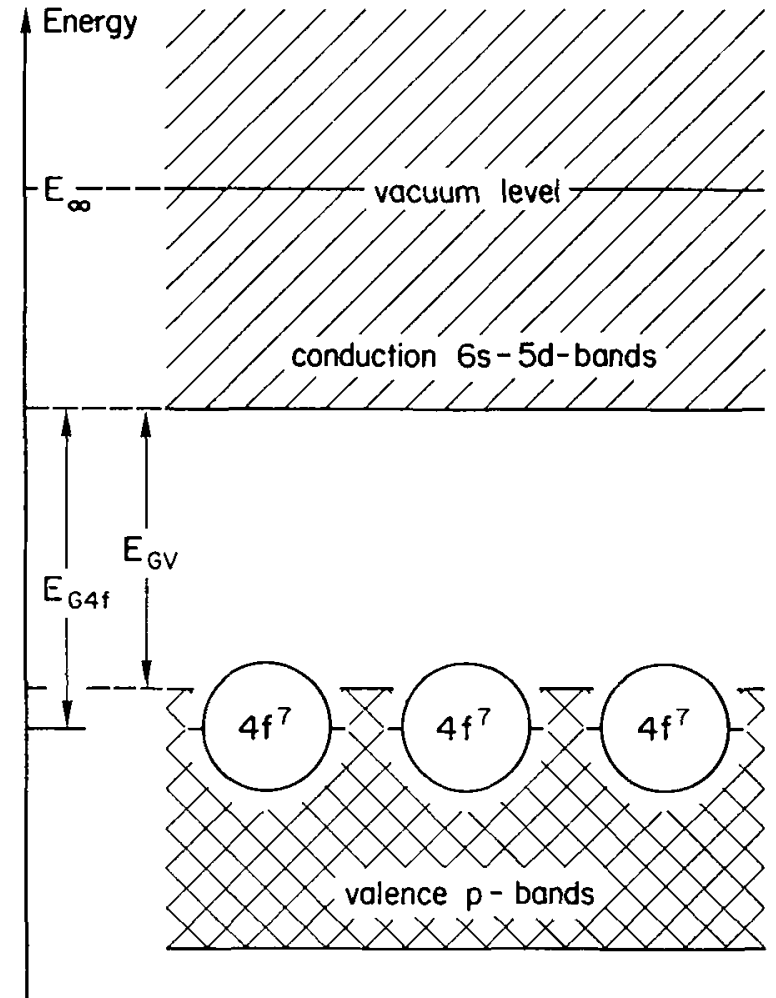

FIG. 10. - Electron states in magnetic semiconductors with strictly localized moments of the half filled $4 \mathrm{f}$-shell.

$\Delta \varphi=\Delta E_{\infty}+\Delta E_{0}$, where $E_{0}$ is the energy of the first occupied electron state $\left(\varphi=E_{\infty}-E_{0}\right)$.

In $\mathrm{GdSb}$ with $E_{\mathrm{GV}}=0$ [20], a large number of states is available at $E_{0}=E_{\mathrm{F}}$ and we can assume $\Delta E_{0}=0$; we obtain $\Delta \varphi=\Delta E_{\infty} . \Delta E_{\mathrm{G}}$ is the energy with respect to the valence band edge of the highest lying new state formed and occupied on disordering. $\Delta E_{\mathrm{G}}$ was calculated from $\Delta \varphi$ with the assumption that $\Delta E_{\infty}=0.3 \mathrm{eV}$ in all substances and using the optical data of Güntherodt, Imboden and Wachter [21]. The results of the measurements and of this calculation are given in table $\mathrm{I}$.

\section{TABLE I}

Data of magnetic semiconductors. a : NaCl-lattice constant, $T_{0}:$ magnetic ordering temperature (*antiferro-, $\mathrm{MnO}$ spin structure). $E_{\mathrm{G} 4 \mathrm{f}}$ and $E_{\mathrm{GV}}$ difference in energy between bottom of conduction bands and $4 f$-levels or valence bands respectively, after G. Güntherodt, D. M. Imboden and P. Wachter, reference [11]. $\varphi$ photoelectric threshold (estimated experimental uncertainty $\pm 0.1 \mathrm{eV}), \Delta \varphi$ change on disordering (uncertainty $\pm 0.15 \mathrm{eV}$ ). $\Delta E_{\mathrm{G}}$ difference in energy between top of valence bands in ordered state and highest occupied levels in disordered state.

$\begin{array}{lcccccc} & \text { EuS } & \text { EuSe } & \text { EuTe } & \text { GdP } & \text { GdAs } & \text { GdSb } \\ a[\AA] & \overline{5} & \overline{6.19} & \overline{6.60} & \overline{5.72} & \overline{5.86} & \overline{6.22} \\ T_{0}[\mathrm{~K}] & 16.5 & 4.6 & 9.8^{*} & 15^{*} & 19^{*} & 28^{*} \\ E_{\mathrm{G} 4 \mathrm{f}}[\mathrm{eV}] & 1.65 & 1.85 & 2.00 & >5 & >5 & >5 \\ E_{\mathrm{GV}}[\mathrm{eV}] & 2.35 & 2.15 & 2.15 & 1.0 & \sim 0.4 & \sim 0 \\ \Phi[\mathrm{eV}] & 4.05 & 4.45 & 4.8 & 3.8 & 4.8 & 4.8 \\ \Delta \Phi[\mathrm{eV}] & 0.8 & 0.6 & 0.6 & 0.5 & 0.3 & 0.3 \\ \Delta E_{\mathrm{G}}[\mathrm{eV}] & \sim 1.2 & \sim 0.6 & \sim 0.5 & \sim 0.2 & \sim 0 & \sim 0\end{array}$


In the model of Mott and Davis [19] the new state should appear at $\Delta E_{\mathrm{G}}=\frac{1}{2} E_{G \mathrm{~V}}$. The present experiments show clearly that $\Delta E_{\mathrm{G}}=$ const. $E_{\mathrm{G} V}$ is not valid. $\Delta E_{\mathrm{G}}$ decreases faster than linearly with $E_{\mathrm{GV}}$.

9. ESP measurements indicate that single occupancy of the states created on disordering is possible due to intra-atomic exchange, and the ferromagnetic coupling of the localized $\mathbf{4 f}$-spins is enhanced. - The most spectacular effects on disordering occur in GdP [5], which exhibits and antiferromagnetic PMC in the I-state and a paramagnetic PMC in the II-state (Fig. 5 and 6). This means that the ferromagnetic part of the exchange has increased relative to the antiferromagnetic one. An increase of the ferromagnetic molecular field on disordering could also be detected in ferromagnetic EuS and metamagnetic EuSe. In contrast to this, EuTe, GdAs and GdSb retained their antiferromagnetic PMC and major changes in the molecular fields were not observed. Enhancement of ferromagnetic exchange thus occurs for the compounds with small lattice constant and a high degree of ionicity of the bonds. The crystals field varies with $\sim a^{-5}$ and it splits the d-states but not the s-states of the cations in such a way that the center of gravity is conserved. Therefore,
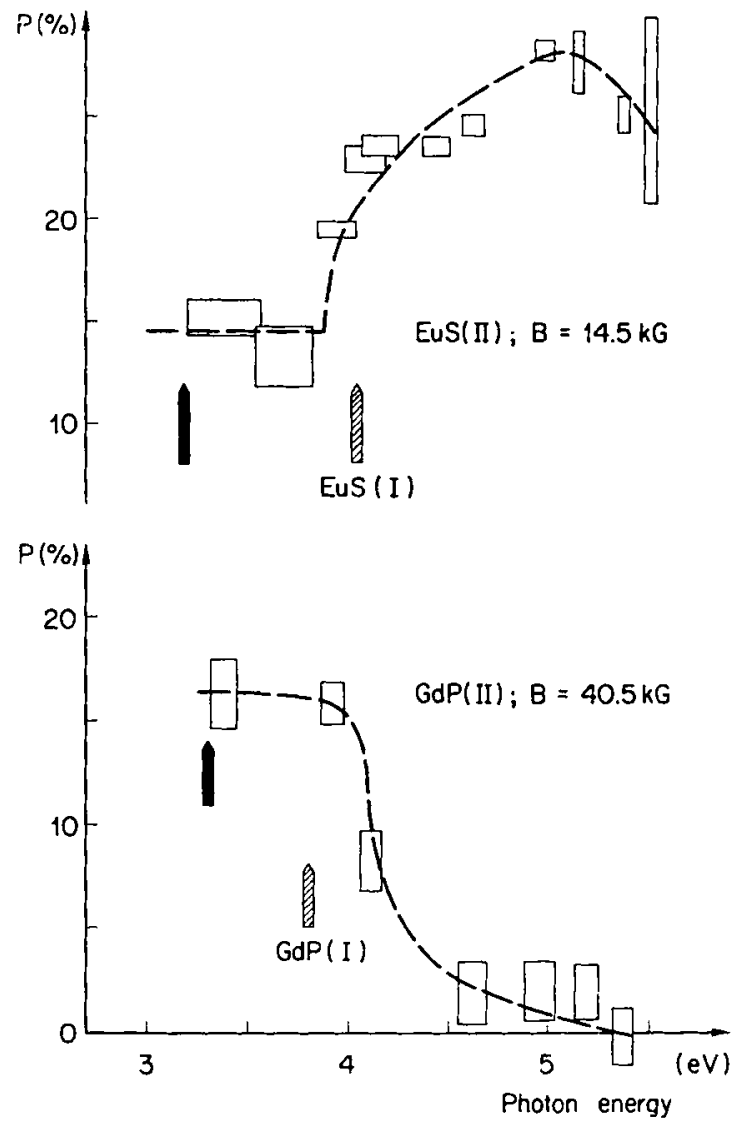

Fig. 11, - Spectra of spin polarization for EuS (II) and GdP (II) ; substrate temperature $4.2 \mathrm{~K}$. Arrows indicate photoelectric threshold, black for disordered (II), hatched for ordered (I) material. the lowest available empty state may have more $\mathrm{d}$-character in the dense lattice and more s-character in the less dense ones. If it is assumed that the lowest states at cation sites can become occupied on disordering, the trend of the observations is readily understood, since occupancy of d-states enhances ferromagnetic exchange.

The SSP's of EuS (II) and GdP (II) are shown in figure 11. With EuS (II), the sharp increase of $P$ at $\hbar \omega \cong 3.8 \mathrm{eV}$ yields the ionization energy of the $4 \mathrm{f}^{7}$-levels, which for EuS (I) is

$$
\varphi=4.05 \pm 0.1 \mathrm{eV} \text {. }
$$

The shift must be attributed to a decrease of $E_{0}$ together with a possible scatter of the $4 f^{7}$-energy position in the disordered lattice. New states which are observed at photon energies below the onset of 4 -transitions cause the remaining part of $\Delta \varphi$ and have a polarization $P^{\prime}$, with $0 \leqq P^{\prime} \neq P_{4 \mathrm{f}}$. The new states are not simply scattered 4 f-states since $P^{\prime}<P_{4 \mathrm{f}}$, and part of them are singly occupied because $P^{\prime} \neq 0$. Thus the singly occupied states postulated to explain the PMC are observed directly in the SSP. The SSP obtained with GdP (II) suggest

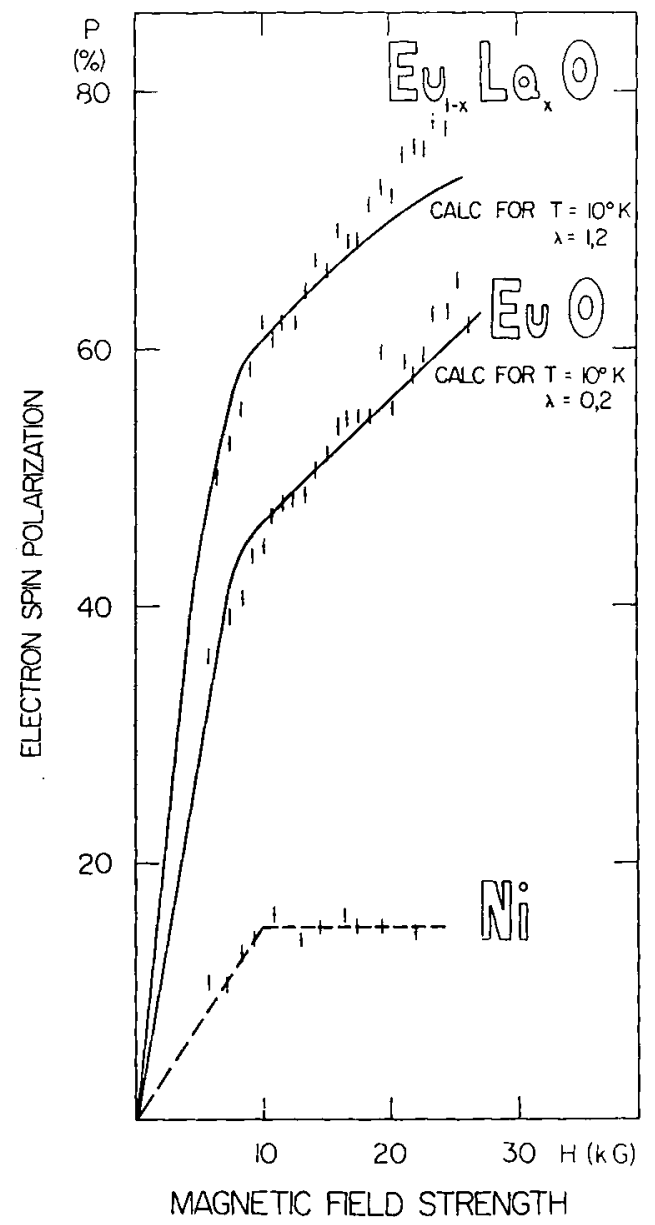

Fig. 12. - PMC's of $\mathrm{EuO}, \mathrm{Eu}_{1-x} \mathrm{La}_{x} \mathrm{O}(x \sim 1$ at $\%)$ and $\mathrm{Ni}$. The full lines are calculated assuming $T=10 \mathrm{~K}$, and the best fit was obtained with the molecular field constants $\lambda$ given in the graph. 
that it is the intra-atomic exchange $J_{\mathrm{df}}$ between $\mathrm{d}$ - and f-orbitals that favors single occupancy. Exchange lifts the spin degeneracy, and the splitting in energy is $2 J_{\mathrm{df}} \mathrm{Ss}=0.7 \mathrm{eV}$ in Eu and Grd. Singly occupied d-states may then exist at least up to $0.7 \mathrm{eV}$ below $E_{0}$ in the SSP of GdP (II).

10. A paramagnetic sheet exists at the surface of the Heisenberg ferromagnet EuO. - - The observation of ESP in photoemission allows one to measure the magnetization in a region very near the surface, by virtue of the small escape depth of the photoelectrons. Experiments have been performed on pure and doped single crystals of EuO [22] (Fig. 12). Fresh (100)-surfaces have been obtained by cleaving in ultrahigh vacuum.

The paramagnetic sheet at the surface explains the fact that magnetic saturation does not occur as seen in figure 12 . The kink at $H=8-10 \mathrm{kG}$ indicates that the bulk however saturates, as it must at temperatures below $T_{\mathrm{c}}$.

The assumption of a paramagnetic surface sheet automatically implies that the exchange coupling of the surface sheet to the bulk is strongly reduced. Assuming a constant $J_{\mathrm{ij}}$, molecular field theory yields the same $T_{c}$ in both the bulk and the surface [23]. A reduced $J_{i j}$ can occur in the outermost atomic layers if the distance between the lattice planes increases. It is known that an increased distance between (100)-planes reduces the ferromagnetic part of the exchange in the europium-chalcogenides [24].

The escape depth of the photoelectrons should be of the order of $50 \AA$ in EuO for photon energies near threshold. This corresponds to 10 lattice constants, and the question arises why one or two para- magnetic layers have such a strong infiuence on the results. It has been shown [25] that electrons travelling from the bulk through the paramagnetic surface sheet into vacuum are very likely to undergo spin exchange collisions with the paramagnetic ions in the surface sheet. By this process, the polarization of the surface sheet is very effectively transferred to the emitted electrons. This is why we see the paramagnetism of the surface despite the fact that only $10 \%$ of the electrons are actually emitted out of the $4 f^{7}$-shell of surface atoms.

11. Doping enhances the molecular field in the paramagnetic surface sheet of EuO. - It remains to explain why the effect of the paramagnetic surface is weaker in the La doped sample as seen from the higher $P$ in figure 12 .

We believe this occurs because the excess electrons introduced by the trivalent La-ions cause an increase of the molecular field in the surface layer and a stronger coupling of the surface to the bulk. This is in analogy with the known fact that in these compounds the Curie-temperature is increased on doping [26] which is attributed to an additional exchange interaction between the localized 4 f-electrons via the introduced electrons.

A paramagnetic surface sheet exists also in thick polycrystalline films of EuS [5].

Acknowledgment. - This work has been supported by the Schweizerischer Nationalfonds. We are extremely grateful to Prof. Dr. G. Busch for his support, encouragement, and interest throughout this work. We are indebted to E. Kaldis and F. Hulliger for the magnetic semiconductor materials.

\section{References}

[1] Mott, N. F., Proc. Roy, Soc., Ser. A 124 (1929) 425.

[2] Busch, G., Campagna, M. and Stegmann, H. C., $J$. Appl. Phys. 41 (1970) 1044.

[3] Raith, W., Atomic Physics (Plenum Press) 1969, p. 389.

[4] Bänninger, U., Busch, G., Campagna, M. and Siegmann, H. C., Pliys. Rev. Lett. 25 (1970) 581.

[5] Busch, G., Campagna, M. and Siegmann, H. C., Int. J. Magnetism 4 (1973) 25 and AIP Conf. Proc. 5 (197l) 575.

[6] Busch, G., Campagna, M., Hulliger, F. and Siegmann, H. C., J. Phys. Chem. Solids 32 (1971) 2173.

[7] Wachter, P. and Wullschleger, J., J. Phys. Chem. Solids 33 (1972) 939.

[8] Alder, H., Campagna, M. and Siegmann, H. C., Phys. Rev. to be published.

[9] Busch, G., Campagna, M. and Siegmann, H. C., Phys, Rev. B 4 (1971) 746

[10] Busch, G., Campagna, M., Pierce, D. T. and Siegmann, H. C., Phys. Rev. Lett. 28 (1972) 611.

[11] Wong, K. C., Wohlfarth, E. P. and Hum, D. M., Phys. Lett. 29A (1969) 2152.

[12] Wohlefakth, E. P., J. Appl. Phys. 41 (1970) 1205.

[13] Andfrson, P. W., Phil. Mag. 24 (1971) 203.

[14] Baltinspikger, W., Helv. Phys. Acta 45 (1972) 203.

[15] Doniach, S., Proc. Int. Conf. on Magnetism and Magnetic
Materials, Chicago 1971, AIP Conf. Proc. 5 (1971) 549.

[16] Brandt, U., Z. Phys. 244 (1971) 217.

[17] Edwards, D. M. and Hertz, J. A., Phys. Rev. Lett. 28 (1972) 1334.

[18] Fadley, C. S. and Wohlfahrth, E. P., Comments on Solid State Phys. 4 (1972) 48.

[19] Motr, N. F. and Davis, E. A., Electronic Processes in non-crystalline materials, (Clarendon Press, Oxford) 1971, and references therein.

[20] Gambino, R. J., Eastman, D. E., McGuire, T. R., Moruzzi V. L. and Grobman, W. D., J. Appl, Phys. 42 (1971) 1468 .

[21] Güntherodt, G., Imboden, D. M. and Wachter, P., Phys. kondens. Mat. 12 (1971) 292.

[22] Sattler, K. and Siegmann, H. C., Phys. Rev. Lett. 29 (1972) 1565.

[23] Mills, D. L., Béal-Monod, M. T. and Weiner, R. A, Phys, Rev. B 5 (1972) 4637 and ref. cited therein.

[24] McGuire, T. R., Argyle, B. E., Schäfer, M. W. and Smart, J. S., J. Appl. Phys. 34 (1963) 1345.

[25] Helman, J. and Siegmann, H. C. (to be published).

[26] Ahn, K. Y. and McGuire, T. R., J. Appl. Phys. 39 (1968) 11. 